\title{
Gengivite necrosante em paciente infantil: relato de caso
}

\author{
Necrotizing gingivitis in a child patient: a case report \\ Gingivitis necrotizante en un paciente infantil: presentación de caso
}

Brenda Karoliny de Araújo Inácio1*, Ana Karina Almeida Rolim², André Higor dos Santos Tavares", Débora Laís de Sousa Castro', Gymenna Maria Tenório Guênes'1, Camila Helena Machado da Costa Figueiredo', João Nilton Lopes de Sousa', Abrahão Alves de Oliveira Filho', Elaine Silva da Penha $^{3}$, Elizandra Silva da Penha'.

\section{RESUMO}

Objetivo: Relatar um caso clínico de gengivite necrosante em uma criança, com diagnóstico clínico de Chikungunya. Detalhamento do caso: Paciente de 8 anos de idade, gênero masculino, que procurou a Clínica Escola de Odontologia da Universidade Federal de Campina Grande relatando "dor na gengiva". Clinicamente foi observada presença de necrose limitada a margem gengival vestibular e nas pontas das papilas interdentais na região antero superior. Foi identificado sinais característicos da doença, como: dor, dificuldade de higienização e alimentação, necrose gengival, odor fétido e candidíase. Foi realizado um tratamento conservador com raspagem e alisamento corononário, limpeza com peróxido de hidrogênio a 10 volumes e prescrição de bochechos com clorexidina a $0,12 \%$ duas vezes ao dia por 15 dias, sem necessidade do uso de antibiótico. Cosiderações finais: A baixa da imunidade por conta do acometimento sistêmico gerado pela Chikungunya foi primordial para o aparecimento da gengivite necrosante. Foi realizado acompanhamento durante quase um mês, onde observou-se o reestabelecimento da saúde periodontal do paciente.

Palavras-chave: Odontologia, Odontopediatria, Doença periodontal.

\begin{abstract}
Objective: To report a clinical case of necrotizing gingivitis in a child, with a clinical diagnosis of Chikungunya. Case details: 8-year-old male patient who visited the Dental School Clinic at the Federal University of Campina Grande reporting "gum pain". Clinically, the presence of necrosis limited to the buccal gingival margin and the tips of the interdental papillae in the upper antero region was observed. Characteristic signs of the disease were identified, such as: pain, difficulty in cleaning and feeding, gingival necrosis, fetid odor and candidiasis. A conservative treatment was performed with scraping and coronary smoothing, cleaning with hydrogen peroxide at 10 volumes and prescription of mouthwashes with $0.12 \%$ chlorhexidine twice daily for 15 days, without the need for antibiotics. Final considerations: Low immunity due to the systemic involvement generated by Chikungunya was essential for the appearance of necrotizing gingivitis. Follow-up was carried out for almost a month, where the patient's periodontal health was reestablished.
\end{abstract}

Keywords: Dentistry, Pediatric dentistry, Periodontal diseases.

\footnotetext{
1 Universidade Federal de Campina Grande (UFCG), Patos - PB. *E-mail: brenda_karoliny@hotmail.com

2 Universidade Estadual da Paraíba (UEPB), Campina Grande - PB.

3 Universidade Federal da Paraíba (UFPB), João Pessoa - PB.
} 


\section{RESUMEN}

Objetivo: Reportar un caso clínico de gingivitis necrotizante en un niño, con diagnóstico clínico de Chikungunya. Detalles del caso: paciente masculino de 8 años que visitó la Clínica de la Facultad de Odontología de la Universidad Federal de Campina Grande y que informó "dolor de encías". Clínicamente, se observó la presencia de necrosis limitada al margen gingival bucal y las puntas de las papilas interdentales en la región antero superior. Se identificaron signos característicos de la enfermedad, tales como: dolor, dificultad en la limpieza y alimentación, necrosis gingival, olor fétido y candidiasis. Se llevó a cabo un tratamiento conservador con escala y suavizado coronario, limpieza con peróxido de hidrógeno a 10 volúmenes y prescripción de enjuagues bucales con clorhexidina al $0,12 \%$ dos veces al día durante 15 días, sin necesidad de antibióticos. Consideraciones finales: la baja inmunidad debido al compromiso sistémico generado por Chikungunya fue esencial para la aparición de gingivitis necrotizante. El seguimiento se llevó a cabo durante casi un mes, donde se restableció la salud periodontal del paciente.

Palabras clave: Odontología, Odontología pediátrica, Enfermedad periodontal.

\section{INTRODUÇÃO}

A falta de higienização e consequente formação de biofilme é uma das principais causas da ausência de saúde periodontal. O seu acúmulo na superfície dentária causa uma série de reações inflamatórias nos tecidos circunvizinhos ao dente, que podem ir desde uma gengivite, até uma periodontite. Existem outros fatores etiológicos que podem contribuir para o aparecimento de patologias do periodonto, como a modificação causada por fatores sistêmicos (ciclo menstrual, puberdade, gravidez...), medicamentos, desnutrição, presença de bactérias, vírus, fungos, entre outras (SPEZZIA S, 2016).

Dentre as enfermidades que acometem o periodonto, as doenças periodontais necrosantes se destacam por representarem a forma mais grave de problema provocado pela ação do biofilme (HERRERA D, et al., 2018; RODRIGUES DM, 2015), e fatores retentores de placa podem contribuir para agravar esses quadros. Fazem parte deste grupo a gengivite necrosante $(G N)$, periodontite necrosante (PN) e estomatite necrosante (EN) e noma (HERRERA D, et al., 2018).

Essas doenças compõem um grupo de condições com etiologia multifatorial e complexa e com o odor fétido como um achado clínico característico (MARTY M, et al., 2016). Apesar de apresentarem baixa prevalência, possuem grande importância, visto que atingem os tecidos periodontais de forma mais rápida e agressiva (HERRERA D, et al., 2014; KWON EY, et al., 2016; BOTERO JE, et al., 2015). A GN é caracterizada pela presença de áreas de ulceração na gengiva marginal com necrose dessas regiões, mas sem envolvimento do periodonto de inserção (KWON EY, et al., 2016).

As doenças periodontais necrosantes em crianças são raras, e quando ocorrem geralmente estão associadas a algum comprometimento sistêmico que resulte em queda da imunidade, possibilitando o aparecimento dos sinais clínicos da doença (MALEK R, et al., 2017). É importante o acompanhamento do cirurgião dentista nesses casos, para uma intervenção precoce, evitando danos maiores ao paciente infantil.

O vírus da Chikungunya foi isolado pela primeira vez em humanos, em 1952, na Tanzânia. Em 2005, ele ressurgiu e no Brasil o primeiro caso foi relatado em 2010 (SÁ PKO, et al., 2017). A região Nordeste apresentou a maior taxa de incidência (56 casos/100.000 habitantes) da doença (BRASIL, 2010). Pessoas acometidas por este vírus geralmente apresentam febre alta, mialgia, dor nas articulações, poliartrite grave e incapacitante, cefaleia, fadiga, náusea, entre outros sintomas. Em relação à cavidade oral, estes pacientes geralmente apresentam gengivites e podem também apresentar sensação de queimação e ulceração da mucosa oral (SÁ PKO, et al., 2017; LEÃO JC, et al., 2018).

Estudo prévio com o intuito de descrever as manifestações orais da Chikungunya, após observação de 97 indivíduos soropositivos para a doença relatou que 55\% destes, apresentaram dor e sangramento gengival, com desconforto durante a mastigação, $42,27 \%$ possuíam gengivite grave e respiração imprópria, que dificultava a realização de atividades rotineiras, e mais de 20\% apresentavam grandes depósitos de placa (KATTI R, et al., 2011). 
Objetivou-se relatar um caso clínico de GN em um paciente infantil acometido por Chikungunya, em uma região endêmica, observando a efetividade do tratamento realizado na fase inicial. Casos como este são raros e importantes para que o profissional tenha maior esclarecimento sobre o desenvolvimento clínico da doença, facilitando o diagnóstico, para possibilitar a realização de um tratamento adequado, no menor tempo possível.

\section{DETALHAMENTO DO CASO}

Trata-se de um caso clínico de um paciente do gênero masculino, 8 anos de idade, que frequenta a Clínica Escola de Odontologia da UFCG desde o ano de 2009. Durante a sua história de atendimento, ele havia realizado tratamentos como: exodontias de elementos decíduos, restaurações, esplintagem do elemento 14 e acompanhamento de aparelho do tipo banda alça para manutenção do espaço para o elemento 34 . Sempre foi caracterizado como sendo um paciente colaborativo e que apresentava higiene bucal variando entre boa e regular. Ao longo dos atendimentos, o acompanhamento da sua condição periodontal foi realizado a partir do Índice de Higiene Bucal (IHB) e do Índice de Sangramento Gengival (ISG) (CARRANZA FA, 1986).

Em setembro de 2013, o seu IHB teve valor de 1,3, que é considerado regular. Já o seu ISG foi bastante elevado, com valor de $57,35 \%$, indicando um alto risco para a saúde do periodonto. Os registros realizados posteriormente nos anos de 2014 e 2015 encontraram valores médios de $\mathrm{IHB}=1,45$, considerado regular, e o ISG $=21,7 \%$, um risco moderado (Tabela 1 ).

Tabela 1 - Dados de todas as avaliações da saúde periodontal pelos índices IHB e ISG durante o período de atendimento do paciente na Clínica de Odontologia da UFCG. Patos- PB, 2016. *Avaliação periodontal realizada após a conclusão do tratamento de GN

\begin{tabular}{ccc}
\hline Atendimento & IHB & ISG (\%) \\
\hline $03 / 09 / 2013$ & 1,3 & 57,35 \\
$19 / 01 / 2014$ & 1,3 & 19,8 \\
$03 / 06 / 2014$ & 1,7 & 19,3 \\
$03 / 11 / 2014$ & 1,3 & 27,1 \\
$28 / 04 / 2015$ & 1,5 & 20,65 \\
$21 / 03 / 2016$ & $0,16^{*}$ & $10,86^{*}$ \\
\hline
\end{tabular}

Fonte: Inacio BK, et al., 2020.

Em fevereiro de 2016, o paciente procurou a Clínica Infantil queixando-se de "dor na gengiva" (SIC). Durante a anamnese, ele relatou estar com dificuldade para se alimentar e realizar higienização dos dentes, devido à dor intensa ao longo dessas atividades, além de ter apresentado quadros febris no dia anterior.

A responsável também relatou que o mesmo apresentava, há aproximadamente 5 dias, dores moderadas de cabeça, articulações e músculos e que, ao procurar atendimento, a criança havia sido diagnosticada clinicamente por um médico, com febre Chikungunya.

Os exames capazes de diagnosticar tal doença não havia sido realizados (sorologia, PCR em tempo real (RT-PCR) e isolamento viral) e o mesmo estava em tratamento (analgésicos) para os sintomas apresentados. Além disso, a criança já estava fazendo uso de Leucogen, medicamento prescrito pelo médico da criança, para estimular o sistema imunológico que estava comprometido.

Ao exame físico foi observada grande presença de biofilme acumulado na superfície dentária de todos os elementos, acompanhada de odor desagradável. A gengiva apresentava-se bastante avermelhada e com sinais de inflamação, além da presença de lesões de candidíase. Na região de pontas de papilas interdentais e gengiva livre, principalmente envolvendo os elementos 11, 12, 21 e 22, era visível presença de áreas necróticas, recobertas por uma fina pseudomembrana (Figura 1 - A).

Depois de concluída a anamnese e exame físico do paciente, levando em consideração os achados clínicos característicos da doença, além da opinião de professores especialistas nas áreas de periodontia e odontopediatria, foi decidido o diagnóstico de Gengivite Necrosante (GN). 
Como tratamento proposto, inicialmente foi realizado desbridamento das áreas necróticas de pontas de papila e gengiva livre, com remoção de pseudomembrana, utilizando Peróxido de Hidrogênio (H2O2) 10 volumes com auxílio de gaze esterilizada, para facilitar o procedimento de limpeza. Remoção do biofilme presente no local, com o auxílio de uma cureta universal do tipo Mccall 13-14. Em seguida foi realizado um bochecho com solução de clorexidina a $0,12 \%$ (marca PerioPlak ${ }^{\circledR}$, lote 0878 ), durante 1 minuto. Ao final do atendimento (Figura 1 - B), foi prescrito ao paciente a realização de bochechos com solução de clorexidina a $0,12 \%$ duas vezes ao dia, durante 1 minuto, por 15 dias; além do antifúngico Daktarin Gel para tratamento da candidíase.

Figura 1 - A) Aspecto clínico inicial do paciente B) Aspecto da gengiva após término do primeiro dia de atendimento. Patos (PB) - 2016.

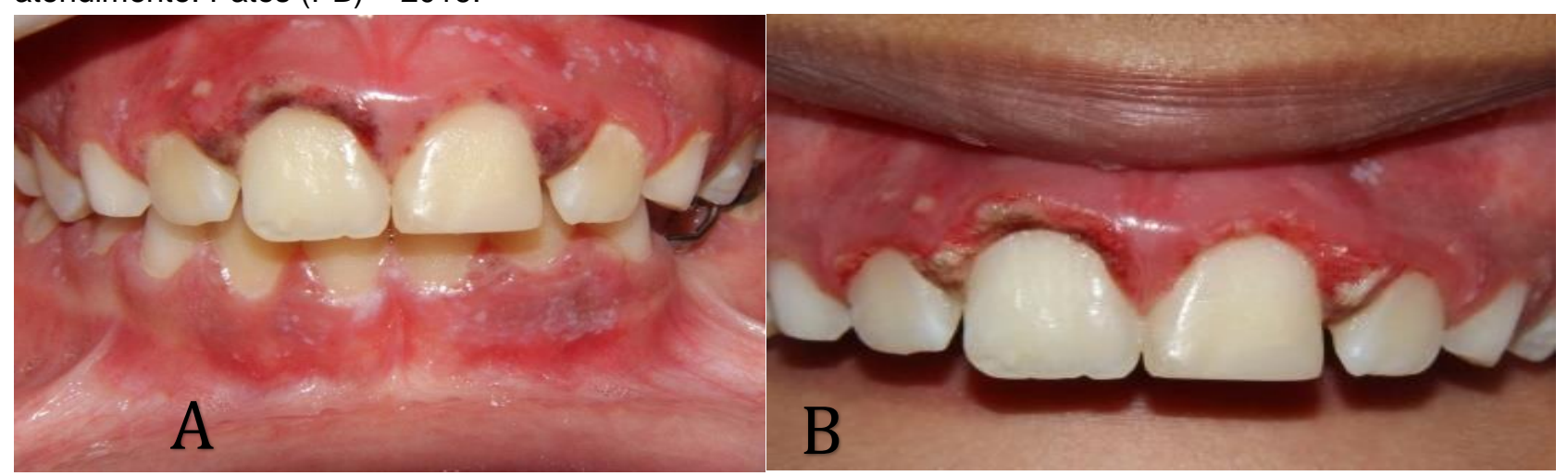

Fonte: Inacio BK, et al., 2020.

Não foi necessária a prescrição de antibiótico para este caso, pois o paciente apresentava como repercussão sistêmica o quadro de febre Chikungunya que já estava em acompanhamento médico. Além disso, foi realizada uma conscientização tanto da criança, como também da sua responsável, sobre a importância de manter uma boa higiene bucal, e o papel fundamental que esses cuidados desempenham sobre o sucesso do tratamento, evitando assim que a doença evolua para apresentações mais graves.

Dois dias após, o paciente retornou para avaliação do caso. Foi observada uma melhora significativa nas áreas necróticas, e de candidíase, mas a gengiva encontrava-se inflamada, além de ainda existir porções de placa bacteriana acumulada sobre as superfícies dentárias. Ele não relatava mais presença de dor durante a escovação ou alimentação. Foi realizada terapia de raspagem com aparelho de ultrassom (marca ORTUS ${ }^{\circledR}$, modelo Bioscaler), com o intuito de facilitar e melhorar o tratamento, poupando tempo nessa etapa do atendimento. $\mathrm{O} \mathrm{H} 2 \mathrm{O} 2$ de 10 volumes também foi utilizado para a limpeza da área, além da realização de bochecho com solução de Clorexidina a $0,12 \%$. Nova orientação de higiene bucal foi feita, reforçando o que foi dito na sessão anterior.

$\mathrm{Na}$ terceira sessão de atendimento, clinicamente foi observada uma melhora significativa na inflamação gengival e houve a completa remissão das lesões necróticas, promovendo o reestabelecimento da saúde periodontal (Figura 2). Nesta fase de manutenção, foi realizada a adequação do meio bucal com profilaxia da região acompanhada de bochechos com solução de clorexidina a $0,12 \%$.

O IHB (Índice de Higiene Bucal) e o ISG (índice de Sangramento Gengival) foram atualizados, e os valores encontrados foram $I H B=0,16$, considerado bom, e o $I S G=10,86 \%$, classificado como de baixo risco para a doença periodontal. Esses valores comprovaram a eficiência do tratamento, mostrando a queda significativa dos índices, após a realização do tratamento para GN. Como sequela da GN, nota-se a irregularidade do contorno gengival (Figura 2), que pode ser corrigido com realização de cirurgia de gengivectomia. 
Figura 2 - Aspecto da gengiva na fase de manutenção após adequação do meio bucal, mostrando o desaparecimento total das áreas necróticas. Patos (PB) - 2016.

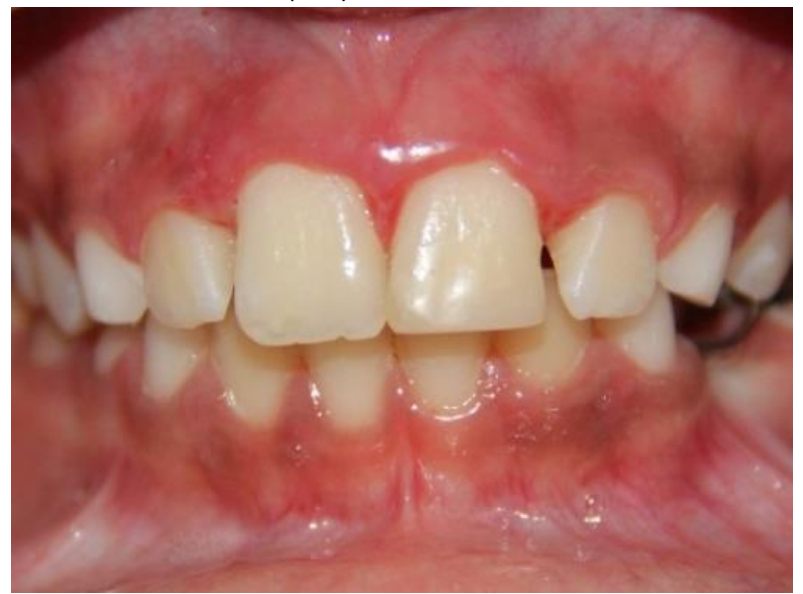

Fonte: Inacio BK, et al., 2020.

\section{DISCUSSÃO}

É demonstrada uma frequente associação entre o aparecimento da GN e o acometimento por alguma enfermidade que acometa a saúde do indivíduo de maneira sistêmica (KUMAR A, et al., 2012). No caso descrito, a doença que atuou facilitando o aparecimento da GN foi a febre Chikungunya.

Devido ao clima propenso para reprodução do mosquito transmissor e a falta de cuidado da população quanto ao acúmulo de água parada em suas casas, o Brasil é um país que apresenta grande potencial para o desenvolvimento da Chikungunya, o que explica o surto ocorrido, que representou um grave problema de saúde pública do país (NUNES MRT, et al., 2015).

Além da dificuldade para o controle dos casos, o diagnóstico destes também era realizado com bastante resistência, pelo fato dos seus sinais clínicos serem semelhantes ao de outras doenças, como a dengue e ZICA. Outro obstáculo encontrado para o diagnóstico foi a dificuldade de realização do teste sorológico para confirmação do quadro. A triagem na rede pública era bem seletiva. Diante disto, o exame sorológico do paciente deste caso não foi realizado, portanto o que havia era um diagnóstico de um médico confirmando a doença pelos sinais clínicos presentes.

As crianças representam um grupo de alto risco para a chikungunya, com manifestações severas e atípicas, com muitos sintomas dermatológicos, como hiperpigmentação, eritema generalizado, edema maculopapular e complicações neurológicas. Algumas alterações orais como aumento no sangramento e inflamação gengival e também presença de estomatite são relatadas (THIBERVILLE S, et al., 2013). Essa ligação entre doenças gengivais necrosantes e condições de comprometimento sistêmico, trazem a necessidade de uma investigação sobre a saúde geral do paciente.

Evidências clínicas mostram que a GN é uma doença debilitante e dolorosa. Quando não tratada, pode evoluir para danos graves aos tecidos periodontais de suporte podendo até provocar a perda de dentes (CARDOSO E, et al., 2019). No paciente infantil esse quadro é ainda mais preocupante, pelo seu limiar de dor ser diferenciado do adulto, o incomodo causado pelas áreas de necrose e descamação vai ser maior, e isso vai interferir na rotina diária da criança, em casa e na escola, refletindo também na vida de toda a família (VIEIRA TR, et al., 2010) Situação que foi relatada no caso, onde o paciente apresentava dificuldade de alimentação e escovação, resultando em grande acúmulo de biofilme na superfície dos seus dentes durante a primeira consulta.

A dor foi o principal motivo para a procura de atendimento odontológico ainda na fase inicial da doença. $O$ diagnóstico é essencialmente clínico, achados como dor, necrose interdental e sangramento, além de odor 
fétido e formação de pseudomembrana podem ser observados (KUMAR A, et al., 2012). Características clínicas que estavam presentes no momento da anamnese, o que facilitou a decisão do diagnóstico.

Para o tratamento, é preconizado seguir o Guideline de terapia periodontal, desenvolvido pela Academia Americana de Periodontia (AAP), também usada pela Academia Americana de Odontopediatria (AAPD), que inclui o desbridamento sob anestesia local e remoção de pseudomembrana usando antisséptico local, realização de prescrição de um antisséptico bucal que seja antibacteriano, e fazer o controle da dor com analgésicos, quando necessário (GREENWEL, 2008).

No caso, não foi necessário a realização de anestesia local, pois inicialmente foi realizado apenas uma neutralização do meio bucal com o $\mathrm{H} 2 \mathrm{O} 2$, para posterior desbridamento, sem realização de raspagem na primeira consulta. No caso relatado para a higienização inicial e desbridamento optou-se pela utilização de um bochecho com solução de clorexidina a $0,12 \%$, para controle dos microrganismos no local, e posteriormente uma limpeza com gaze esterilizada embebida em $\mathrm{H} 2 \mathrm{O} 2$, para remoção da pseudomembrana.

A maioria dos autores recomendam bochechos com clorexidina a $0,12 \%$ duas vezes ao dia (ATOUT RN e TODESCAN S, 2013; BLAIR FM e CHAPPLE IL, 2014; SANGANI I, et al., 2013). Mas há autores que preferem usar o peróxido de hidrogénio diluído em água (HODGDON A, 2016). Na clínica de odontologia da UFCG, realiza-se a prescrição de bochechos com solução de clorexidina a $0,12 \%$, durante 1 minuto, duas vezes ao dia, por 15 dias. Para a realização da raspagem de remoção do cálculo, além da utilização de curetas, é cada vez mais comum na prática clínica o uso de instrumentos como o ultrassom e laser (KINA JR, et al. 2014; KRISHNA R e STEFANO JA 2016). O ultrassom foi utilizado na segunda sessão de atendimento, para acelerar a raspagem, agilizando $o$ tratamento.

Como o paciente já estava sendo acompanhado por um médico para tratamento da Chikungunya, uma doença relativamente nova e com poucos estudos sobre as suas reais consequências, houve um consenso entre os profissionais envolvidos no tratamento da GN sobre a não necessidade de utilização de antibióticoterapia sistêmica.

Mesmo com os inúmeros achados clínicos que são característicos da GN, ainda existe uma dificuldade entre os clínicos para a realização do diagnóstico (MICHEL M, SOLEDADE KR e AZOUBEL EMCFA, 2012). Neste caso, a periodontia e a odontopediatria se complementaram, o que facilitou o diagnóstico da doença. Outro fato primordial para o sucesso do tratamento foi a interação da tríade: paciente, profissional, responsável. $\mathrm{O}$ tratamento periodontal, pela importância da manutenção da higienização adequada para o seu sucesso, pede uma atenção especial dos pais quanto aos hábitos da criança durante a sua rotina diária.

Considerando-se o risco de evolução para extensas doenças necrosantes, está comprovado que uma interceptação eficaz nos primeiros dias de infecção da GN é de extrema importância. Vários estudos afirmam que, quando o tratamento correto é seguido, ocorre a remissão das áreas necróticas por completo, e a saúde periodontal é recomposta (NUNES MRT, 2015; TAUIL PL, 2014; THIBERVILLE S, et al., 2013; VOLCHANSKY A, et al., 2015).

\section{CONSIDERAÇÕES FINAIS}

Com base no exposto, pode-se identificar que existe uma relevante associação entre a Chikungunya e sua repercussão na cavidade oral. Enfatiza-se a importância do conhecimento do cirurgião-dentista acerca das lesões que podem acometer a cavidade oral de crianças, em especial, as com comprometimento sistêmico. O diagnóstico preciso e o tratamento adequado da GN em sua fase inicial neste caso, foram cruciais para a recuperação periodontal do paciente, proporcionando o total desaparecimento dos sinais clínicos da doença, além do reestabelecimento da saúde periodontal da criança, comprovada pelos dados obtidos nos índices de IHB e ISG. Além disso, evidencia-se a necessidade de proservação até a completa recuperação do paciente, em casos como este. 


\section{REFERÊNCIAS}

1. ATOUT RN, TODESCAN S. Managing patients with necrotizing ulcerative gingivitis. J Can Dent Assoc, 2013;79(46):1-4.

2. BLAIR FM, CHAPPLE IL. Prescribing for periodontal disease. Prim Dent J, 2014; 3(4):38-43.

3. BOTERO JE, et al. Periodontal disease in children and adolescents of Latin America. Periodontology 2000, 2015; 67(1):34-57.

4. BRASIL. Ministério da Saúde. Secretaria da Vigilância em Saúde, Departamento de Vigilância Epidemiológica. Casos importados da Febre Chikungunya no Brasil. Brasília: Ministério da Saúde, 2010.

5. CARDOSO E, et al. Gengivite necrosante: Uma abordagem literária. Revista de Odontologia Contemporânea. 2019; 3(2):71-71.

6. CARRANZA FA. Peridontología clínica de Glickmam. 6ta ed. México: Nueva editorial Interamericana; 1986;6:405-490.

7. GREENWELL H. Guideline for periodontal therapy. Pediatric dentistry, 2008; 30(7):248-52.

8. HERRERA D, et al. Acute periodontal lesions (periodontal abscesses and necrotizing periodontal diseases) and endo-periodontal lesions. Journal of clinical periodontology, 2018; 45(20):78-94.

9. HERRERA D, et al. Periodontal lesions. Periodontol 2000, 2014; 65(1)149-177.

10. HODGDON A. Dental and related infections. Emerg Med Clin North Am, 2013;31(2):465-80.

11. KATTI R, et al. Impact of Chikungunya virus infection on oral health status: an observational study. Indian J Dent Res, 2011; 22(4): 613.

12. KINA JR, et al. Periodontite ulcerativa necrosante: tratamento físico da superfície radicular com Erbium YAG laser. Arch Health Invest, 2014; 3(3):33-38.

13. KRISHNA R, STEFANO JA. Ultrasonic vs. Hand instrumentation in periodontal therapy: clinical outcomes. Periodontology 2000, 2016; 71(1):113-127.

14. KUMAR A, et al. Periodontal diseases in children and adolescents: a clinician's perspective part 2. Dent Update, 2012; 39(9):639-42. 645-6, 649-52.

15. KWON EY, et al. Effective Management of Acute Necrotizing Ulcerative Gingivitis with Proper Diagnosis and Immediate Treatment. Journal of Korean Dental Science, 2016; 9(2):81-89.

16. LEÃO JC, et al. Chikungunya fever: General and oral healthcare implications. Oral Diseases, 2018; 24(12):233-237.

17. MALEK R, et al. Necrotizing ulcerative gingivitis. Contemporary clinical dentistry, 2017; 8(3): 496-500.

18. MARTY M, et al. Necrotizing Periodontal Diseases in Children: A Literature Review and Adjustment of Treatment. Journal of tropical pediatrics, 2016; 62(4):331-337.

19. MICHEL M, et al. Doenças periodontais necrosantes e uso de antimicrobianos como terapia adjuntarevisão da literatura. Braz J Periodontol-March, 2012; 22(01).

20. NUNES MRT, et al. Emergence and potential for spread of Chikungunya virus in Brazil. BMC Medicine, 2015;13(1):102.

21. RODRIGUES DM. Severe localized aggressive periodontitis combined with necrotizing ulcerative gingivitis: a 10-year follow-up of a multidisciplinary treatment. Perionews, 2015; 9(4):301-306.

22. SÁ PKO, et al. Chikungunya virus infection with severe neurologic manifestations: report of four fatal cases. Revista da Sociedade Brasileira de Medicina Tropical, 2017; 50(2):265-268.

23. SANGANI I, et al. Necrotizing ulcerative gingivitis and the orthodontic patient: A case series. Journal of orthodontics, 2013;40(1);77-80.

24. SPEZZIA S. Inter-relação entre hormônios sexuais e doenças periodontais nas mulheres. Braz J Periodontol, 2016; 26(2):40-47.

25. TAUIL PL. Condições para transmissão da febre do vírus chikungunya. Epidemiologia e Serviços de Saúde, 2014; 23(4):773-774.

26. THIBERVILLE S, et al. Chikungunya fever: Epidemiology, clinical syndrome, pathogenesis and therapy. Antiviral Research. 2013;99(3):345-370.

27. VIEIRA TR, et al. Alterações periodontais associadas às doenças sistêmicas em crianças e adolescentes. Revista Paulista de Pediatria. 2010;28(2):237-243.

28. VOLCHANSKY A, et al. Thirty six years of periodontal care: a case report. Jornal Dental Sul Africano, 2015; 70(5):210-212. 